\title{
Acadêmicos de saúde atuando em escolas públicas, Manaus - AM: relato de experiência
}

\section{Health students working in public schools, Manaus - AM: experience report}

DOI: $10.46919 / \operatorname{archv1n6-003~}$

Recebimento dos originais: 01/09/2020

Aceitação para publicação: 30/10/2020

\section{Renato da Cunha Araújo}

Médico formado pela Universidade do Estado do Amazonas (UEA). Pós-graduando do Curso de Especialização em Medicina de Família e Comunidade (Escola de Saúde Pública de Manaus / UEA). Instituição: Universidade do Estado do Amazonas

endereço: Av. Carvalho Leal, 1777 - Cachoeirinha, Manaus - AM, CEP 69065-001, Escola Superior de

Ciências da Saúde ESA/UEA).

E-mail: rhen_ca@hotmail.com

\section{Priscila Soares de Souza}

Enfermeira formada pela Universidade do Estado do Amazonas (UEA). Especialização em Urgência e Emergência (Faculdade Delta). Universidade do Estado do Amazonas.

Instituição: Universidade do Estado do Amazonas

endereço: Av. Carvalho Leal, 1777 - Cachoeirinha, Manaus - AM, CEP 69065-001, Escola Superior de

Ciências da Saúde ESA/UEA).

E-mail: pri_cilinhaS2@hotmail.Com

\section{Josiani Nunes do Nascimento}

Enfermeira formada pela Universidade Federal do Amazonas (UFAM). Especialização em Unidade de Terapia Intensiva (UFAM), Urgência \Emergência (UFAM) e em Educação Profissional na Área de Saúde: Enfermagem (FIOCRUZ), docente da UEA. Instituição: Universidade do Estado do Amazonas

endereço: Av. Carvalho Leal, 1777 - Cachoeirinha, Manaus - AM, CEP 69065-001, Escola Superior de Ciências da Saúde ESA/UEA).

E-mail: josiani.nunes@ig.com.br

\section{Cássia Rozária da Silva Souza}

Enfermeira formada pela Universidade Federal do Amazonas (UFAM). Especialização em Administração Hospitalar e Unidades de Saúde; em Enfermagem Obstétrica, em Formação Pedagógica em SaúdePROFAE; em Geografia em Saúde. Mestre em Ciências do Ambiente. Docente na Universidade do Estado do Amazonas (UEA). Doutorado em Enfermagem pelo Programa de Doutorado Interinstitucional da Universidade Federal de Santa Catarina e Universidade do Estado do Amazonas. Instituição: Universidade do Estado do Amazonas

endereço: Av. Carvalho Leal, 1777 - Cachoeirinha, Manaus - AM, CEP 69065-001, Escola Superior de Ciências da Saúde ESA/UEA).

E-mail: silvarozaria@ig.com.br 


\title{
Nilcéia Aparecida Mota Marques
}

Médica formada pela Universidade do Estado do Amazonas (UEA). Especialização em Infectologia (FMT-HVD). Especialização em Vigilância Sanitária pela FIOCRUZ (1998), Especialização em Segurança do Paciente, pela FIOCRUZ (2015), Mestrado em Saúde e Gestão do Trabalho pela

Universidade do Vale do Itajaí - SC (2005).

Instituição: Universidade do Estado do Amazonas

endereço: Av. Carvalho Leal, 1777 - Cachoeirinha, Manaus - AM, CEP 69065-001, Escola Superior de Ciências da Saúde ESA/UEA).

E-mail: nilceiamota@hotmail.com

\section{RESUMO}

A promoção da saúde consiste em capacitar a população para melhorar suas condições de saúde e aumentar o seu controle sobre as mesmas. Além disso, é definida como uma combinação de apoios educacionais e ambientais que visam a divulgação de informações acerca de hábitos de vida saudáveis, repassando conhecimentos e habilidades para o autocuidado da saúde. Este estudo tem por finalidade descrever as atividades teóricas e práticas desenvolvidas na disciplina "Atenção Integral à Saúde", pelos acadêmicos de Enfermagem, Medicina e Odontologia, da Universidade do Estado do Amazonas, enfatizando o trabalho interdisciplinar a serviço da comunidade. A disciplina do $1^{\circ}$ período almeja inserir o discente na realidade da saúde regional e nacional, isto é, fazendo-o ser capaz de compreender o Sistema Único de Saúde-SUS/Estratégia Saúde da Família. A parte teórica baseou-se em aulas expositivas, investigação científica, problematização e debate de diversos temas relacionados à saúde pública. Quanto à prática, houve apresentação dos discentes em escolas da rede pública de ensino, preparada sob orientação, divisão dos grupos, discussão das temáticas e confecção de recursos didáticos contextualizados para as respectivas faixas etárias e temáticas. As equipes apresentaram atividades didáticas-lúdicas para cerca de 360 alunos de diferentes séries, permitindo a interação e integralidade dos cursos. O foco principal apoiou-se na orientação educacional e prevenção de doenças e agravos. Após as práticas, as equipes entregam um relatório descrevendo suas reflexões e sugestões acerca da atividade executada, introduzindo, desta maneira, o senso crítico-científico nos acadêmicos participantes. Nota-se que esta metodologia configura uma forma de praticar a interdisciplinaridade - discentes, aprendem a trabalhar de forma humanizada e em grupo; docentes, porque se vêem compelidos a ampliar o conhecimento; a instituição de ensino, por se inserir na proposta pedagógica da disciplina; a comunidade infanto-juvenil, por oportunizar e integrar-se as dinâmicas educacionais em prol da promoção da saúde, tornando-se multiplicadores.

Palavras-chave: Atenção Integral a Saúde, Integralidade, Promoção da Saúde, Saúde Coletiva, Educação em Saúde.

\begin{abstract}
Health promotion consists of training the population to improve their health conditions and increase their control over them. In addition, it is defined as a combination of educational and environmental support aimed at disseminating information about healthy living habits, passing on knowledge and skills for selfcare of health. This study aims to describe the theoretical and practical activities developed in the discipline "Comprehensive Health Care", by Nursing, Medicine and Dentistry students, from the State University of Amazonas, emphasizing the interdisciplinary work at the service of the community. The discipline of the 1st period aims to insert students in the reality of regional and national health, that is, making them able to understand the Unified Health System-SUS / Family Health Strategy. The theoretical part was based on lectures, scientific research, problematization and debate on various topics related to public health. As for the practice, there was a presentation by students in public schools, prepared under guidance, division of groups, discussion of the themes and preparation of contextualized teaching resources for the respective age and thematic groups. The teams presented didactic-ludic activities to
\end{abstract}


about 360 students from different grades, allowing the interaction and completeness of the courses. The main focus was based on educational guidance and prevention of diseases and injuries. After the practices, the teams deliver a report describing their reflections and suggestions about the activity performed, introducing, in this way, the critical-scientific sense in the participating academics. It is noted that this methodology is a way of practicing interdisciplinarity - students, learn to work in a humanized and group way; teachers, because they are compelled to expand knowledge; the educational institution, for being inserted in the pedagogical proposal of the discipline; the child and youth community, for providing opportunities and integrating educational dynamics in favor of health promotion, becoming multipliers.

Keywords: Comprehensive Health Care, Integrality, Health promotion, Collective Health, Health education.

\section{INTRODUÇÃO}

A promoção da saúde consiste em capacitar a população para melhorar suas condições de saúde e aumentar o seu controle sobre as mesmas. Além disso, é definida como uma combinação de apoios educacionais e ambientais que visam a divulgação de informações acerca de hábitos de vida saudáveis, repassando conhecimentos e habilidades para o autocuidado da saúde ${ }^{1-2}$.

O Campo da Saúde Coletiva possibilita o exercício do paradigma da Interdisciplinaridade, com a construção de saberes e práticas de disciplinas e subdisciplinas novas, dispondo métodos e conteúdos teóricos próprios, voltados para a saúde pública, e adaptados à populações específicas - considerando aspectos socioculturais, políticos, econômicos e geográficos ${ }^{3}$.

Atividades direcionadas as coletividades, sem delimitar grupos quanto a doença, condição ou risco, promovem qualidade de vida à população, tendo um maior alcance de seus benefícios. Debates e reflexões acerca da Promoção da Saúde começaram desde os anos de 1970, e foram marcados na Carta de Ottawa (Ottawa Charter for Health, 1987), sendo um ponto referencial na elaboração de conceitos e princípios em saúde. Desde então, são estudadas e preconizadas ideias como a responsabilização do setor público em propor políticas saudáveis; estimulação às ações comunitárias; cuidados com o meio ambiente; aprimoramento de habilidades pessoais; e reorientação dos serviços de saúde. Tais pressupostos apresentam aspectos social, transformador e questionador, no âmbito individual e coletivo, enfatizando a participação e o controle social como essenciais na implementação de ações de Educação em Saúde ${ }^{4-5}$.

\section{OBJETIVOS}

Descrever as atividades teóricas e práticas desenvolvidas na disciplina "Atenção Integral à Saúde", pelos acadêmicos de Enfermagem, Medicina e Odontologia, da Universidade do Estado do Amazonas, enfatizando o trabalho interdisciplinar a serviço da comunidade. 


\section{METODOLOGIA}

Trata-se de um estudo descritivo e qualitativo. A disciplina do $1^{\circ}$ período almeja inserir o discente na realidade da saúde regional e nacional, isto é, fazendo-o ser capaz de compreender o Sistema Único de Saúde-SUS/Estratégia Saúde da Família.

A parte teórica baseou-se em aulas expositivas, investigação científica, problematização e debate de diversos temas relacionados à saúde pública. Quanto à prática, houve apresentação dos discentes em escolas da rede pública de ensino, preparada sob orientação, divisão dos grupos, discussão das temáticas e confecção de recursos didáticos contextualizados para as respectivas faixas etárias e temáticas (drogas lícitas e ilícitas, higiene, nutrição regional, doenças endêmicas e saúde ambiental).

\section{RESULTADOS}

As equipes apresentaram atividades didáticas-lúdicas para cerca de 360 alunos de diferentes séries do Ensino Fundamental e Médio, permitindo a interação e integralidade dos cursos. O foco principal apoiou-se na orientação educacional e prevenção de doenças e agravos.

Após as práticas, as equipes entregam um relatório descrevendo suas reflexões e sugestões acerca da atividade executada, introduzindo, desta maneira, o senso crítico-científico nos acadêmicos participantes.

Os discentes mencionaram terem sido estimulados diante do desafio lançado e estarem com um sentimento de satisfação quanto ao serviço prestado para a sociedade. No tocante aos gestores das escolas atendidas, solicitaram que tal atividade se tornasse periódica.

\section{DISCUSSÃO}

Ações no âmbito da Educação em Saúde, como a apresentada neste relato de experiência, são práticas potenciais de promoção da saúde. Conforme Maciel, et al (2006), as atividades de promoção da saúde no ambiente escolar, amplia a evolução de ideias, habilidades, conhecimentos, criatividade, capacidade de tomar decisões, adquirir hábitos saudáveis e desenvolvimento de ambientes estimuladores de saúde; destacando-se as ações educativas e sanitárias adaptadas aos participantes de uma determinada comunidade local.

Assim como ressaltado por Queiroz e Jorge (2006), em busca de uma sociedade melhor, a formação dos acadêmicos da área de saúde deve englobar o desenvolvimento de senso crítico, de maneira que o estudante - futuro profissional de saúde - torne-se capaz de identificar e atuar em um cenário desumanizante. Assumindo a responsabilidade em observar, comparar, avaliar, decidir e intervir, na condição de saúde da comunidade, onde se encontra inserido. 
Os participantes praticaram reflexões críticas e síntese analítica da situação de saúde, da realidade vivenciada, gerando conceitos de saúde e saneamento, de acordo com

Tal experiência provoca uma crítica reflexiva quanto ao modelo tradicional de formação dos profissionais de saúde, empenhado pelas instituições de ensino, isto é, baseado em uma forma pedagógica biologista, compartimentada e medicalizante, não existindo interação real do ensino com as questões sociais de saúde. Assim, devendo-se buscar não somente o desenvolvimento da relação alunoconhecimento, como também "para que" e "para quem será aplicada" esta habilidade de crítica e de construção dos saberes; sendo tal capacidade do acadêmico aplicada a serviço dos interesses maiores da comunidade, conforme interpretado por CHIRELLI e MISHIMA (2002), e Amaral et al (2014).

Ressaltando as reflexões feitas por Mello e Colaboradores (1998), uma perspectiva inovadora em saúde pública, cuja abordagem envolve a comunidade, grupos e indivíduos exercendo cidadania e participando, decisivamente, das políticas públicas de saúde, faz-se necessária diante do cenário desafiador imposto pelos princípios preconizados pelo Sistema Único de Saúde, onde se almeja levar o acesso aos serviços de saúde à todos, de forma adaptada às peculiaridades de cada cidadão e considerando-o em sua completude física, mental, social, econômica e cultural.

\section{CONCLUSÃO}

É essencial reconhecer a Educação em Saúde como um possível instrumento de construção da articulação ensino - comunidade - serviço de saúde. Além disso, as ações efetivadas através de palestras e rodas de discussão protagonizadas pelos discentes universitários e os alunos da rede pública de ensino, aperfeiçoam a confiança e o vínculo estabelecido entre usuários e profissionais participantes do Sistema Único de Saúde. O desenvolvimento da prática educativa com universitários e alunos da rede pública tornam ambos sensibilizados à meta de busca da melhoria das condições de saúde vigentes em seu meio.

Podemos praticar a interdisciplinaridade - discentes, aprendem a trabalhar de forma humanizada e em grupo; docentes, porque se vêem compelidos a ampliar o conhecimento; a instituição de ensino, por se inserir na proposta pedagógica da disciplina; a comunidade infanto-juvenil, por oportunizar e integrar-se as dinâmicas educacionais em prol da promoção da saúde, tornando-se multiplicadores. 


\section{REFERÊNCIAS}

1. CECÍLIO, L.C. As necessidades de saúde como conceito estruturante na luta pela integralidade e eqüidade na atenção em saúde. In: Pinheiro R, Mattos RA orgs. Os sentidos da integralidade na atenção e no cuidado à saúde. 4. ed. Rio de Janeiro: IMS-UERJ/CEPESC/ABRASCO; 2006.

2. MACIEL, E. L. N., et al. Projeto aprendendo saúde na escola: a experiência de repercussões positivas na qualidade de vida e determinantes da saúde de membros de uma comunidade escolar em Vitória, Espírito Santo. Ciência \& Saúde Coletiva 2010; 15(2): 389-96.

3. LUZ, M.T. Complexidade do Campo da Saúde Coletiva: multidisciplinaridade, interdisciplinaridade e transdisciplinaridade de saberes e práticas - análise sócio-histórica de uma trajetória paradigmática. Saúde e Sociedade, v. 18, n. 2, p. 304-311, 2009.

4. QUEIROZ, M.V; JORGE, M.S. Estratégias de educação em saúde e a qualidade do cuidar e ensinar em pediatria: a interação, o vínculo e a confiança no discurso dos profissionais. Revista Interface Comunicação, Saúde, Educação 2006; 10(19): 117-30.

5. MEllo, D. A.; ROUQUAYROL, M. Z.; BENTO, L. F.; et al. Promoção à saúde e educação: diagnóstico de saneamento através da pesquisa participante articulada à educação popular. Cad Saúde Pública 1998; 14:583-95.

6. CHIRELLI, M.Q.; MISHIMA, S. M. O processo de formação do enfermeiro crítico reflexivo na visão dos estudantes do curso de enfermagem da FAMEMA. 2002. Ribeirão Preto; 2002. Doutorado [Tese] Escola de Enfermagem de Ribeirão Preto, Universidade de São Paulo.

7. AMARAL, M.C.S.; PONTES, A.G.V.; SILVA, J. V. O ensino de Educação Popular em Saúde para o SUS: experiência de articulação entre graduandos de Enfermagem e Agentes Comunitários de Saúde. Interface, v. 18, Suppl 2, p. 1547-1558, 2014. 